\title{
Intraamygdala Infusion of Fibroblast Growth Factor 2 Enhances Extinction and Reduces Renewal and Reinstatement in Adult Rats
}

\author{
Bronwyn M. Graham and Rick Richardson \\ School of Psychology, University of New South Wales, Sydney, New South Wales 2052, Australia
}

Systemic fibroblast growth factor 2 (FGF2) has been shown to enhance extinction of conditioned fear and attenuate subsequent relapse in developing rats. However, it is not clear whether FGF2 has the same effect in adult rats, and furthermore, the neuroanatomical locus of the effect of FGF2 on extinction is unknown. These experiments examined the effect of $200 \mathrm{ng}$ of FGF2, infused bilaterally into the basolateral complex of the amygdala (BLA), on the extinction of conditioned fear in adult rats. Experiment 1 confirmed that intra-BLA FGF2 significantly enhances extinction recall in adult rats, and extinction training is necessary for this effect to occur (FGF2 did not reduce conditioned freezing in the absence of extinction training). In Experiments 2 and 3, vehicle-treated rats were given four times the amount of extinction training as FGF2-treated rats to equate the strength of extinction between groups. In Experiment 2, rats were tested in both the extinction training context and the conditioning context to examine the effect of FGF2 on renewal of fear. In Experiment 3, the FGF2-treated rats and one-half of the vehicle-treated rats received a single unsignaled shock before test to examine the effect of FGF2 on reinstatement of fear. In both procedures, FGF2 administered immediately after extinction training significantly reduced relapse at test. These results support a growing body of evidence that FGF2 may be a potentially useful pharmacological adjunct to exposure-based therapies for anxiety disorders.

\section{Introduction}

Exposure therapy is based on extinction, a laboratory procedure in which conditioned fear responses decline as a result of repeated exposure to a feared conditioned stimulus (CS). Exposure therapy is the most widely used treatment for anxiety disorders, and while successful for many, it is associated with a high treatment dropout rate, and a significant proportion fail to maintain treatment gains (McNally, 2007; Hofmann and Smits, 2008). A potential solution is to develop pharmacological adjuncts that enhance the learning processes underlying fear extinction (Graham et al., 2011). Although several agents have proved successful in enhancing extinction, few have been demonstrated to reduce subsequent relapse of fear. One exception is the neurotrophic factor fibroblast growth factor 2 (FGF2). Systemic injections of FGF2 before or immediately after fear extinction enhance extinction recall in postnatal day 23 (P23) rats, and furthermore, treatment with FGF2 renders rats significantly less susceptible to common forms of relapse including reinstatement (i.e., stress-induced) and re-

Received June 14, 2011; revised July 28, 2011; accepted Aug. 6, 2011.

Author contributions: B.M.G. and R.R. designed research; B.M.G. performed research; B.M.G. and R.R. analyzed data; B.M.G. and R.R. wrote the paper.

This work was supported by Australian Research Council Discovery Project Grant DP0985554 (R.R.). We thank Stella Li for her contribution in teaching B.M.G. the surgical techniques used in these experiments.

The authors declare no competing financial interests.

Correspondence should be addressed to Bronwyn M. Graham, Department of Psychiatry, Harvard Medical

School and Massachusetts General Hospital, 149 13th Street, CNY 2614, Charlestown, MA 02129. E-mail: bmgraham@nmr.mgh.harvard.edu.

DOI:10.1523/JNEUROSCI.3014-11.2011

Copyright $\odot 2011$ the authors $\quad 0270-6474 / 11 / 3114151-07 \$ 15.00 / 0$ newal (i.e., relapse associated with a change in test context) (Graham and Richardson, 2009, 2010). A recent study suggests that FGF2 may enhance fear extinction via partially erasing the original fear memory (Graham and Richardson, 2011). Together, these findings make FGF2 an attractive candidate for a novel pharmacological adjunct to exposure therapy.

Despite its promise, there are several questions regarding the effect of FGF2 on extinction. Previous investigations of the effects of FGF2 on extinction have involved developing rats, and therefore it is unknown whether FGF2 has similar effects on extinction in adults. This issue deserves investigation. The pattern of FGF2 expression across the life span has not been well documented; however, it has been reported that endogenous FGF2 declines in middle-aged rats (Rai et al., 2007) and that FGF2 is differentially expressed both spatially and temporally across various stages of early development in P0-P30 rats (Monfils et al., 2006). Thus, the reported effects of systemic FGF2 on fear extinction in P23 rats may not occur in adult rats. Furthermore, previous experiments have involved systemic injections of FGF2, and it is unknown whether FGF2 acts centrally to enhance extinction, and if so, which neural structures it targets. In the present experiments, we begin to address these questions by examining the effect of infusions of FGF2 into the basolateral complex of the amygdala (BLA) on extinction recall, renewal, and reinstatement in adult rats. We reasoned that the amygdala is the most likely target of FGF2 given that (1) the amygdala is a critical structure in the neural circuitry of extinction and (2) the amygdala is thought to be the structure in which the original fear conditioning memory is stored (LeDoux, 2000). 


\section{Materials and Methods}

Subjects and surgery

Male Sprague Dawley rats (250-350 g; P90) obtained from a commercial supplier (Animal Resources Centre, Perth, Western Australia, Australia) were used. Rats were housed in groups of eight in plastic cages (67 $\mathrm{cm}$ long $\times 30 \mathrm{~cm}$ wide $\times 22 \mathrm{~cm}$ high) in a colony room maintained at $20-22^{\circ} \mathrm{C}$ on a $12 \mathrm{~h} \mathrm{light/dark} \mathrm{cycle} \mathrm{(lights} \mathrm{on} \mathrm{at} \mathrm{7:00} \mathrm{A.M.).} \mathrm{Food} \mathrm{and}$ water were available ad libitum. All animals were treated according to the principles of animal use outlined in Australian Code of Practice for the Care and Use of Animals for Scientific Purposes, Seventh Edition (2004), and all procedures were approved by the Animal Care and Ethics Committee of the University of New South Wales. Before surgery, rats were anesthetized with isoflurane (Laser Animal Health) mixed with oxygen. Anesthetized rats were then mounted on a stereotaxic apparatus (Kopf Instruments), and 26 gauge guide cannulas (Bioscientific) were implanted bilaterally in the BLA through holes drilled in the skull (anteroposterior, $-2.8 \mathrm{~mm}$; mediolateral, $\pm 5 \mathrm{~mm}$; dorsoventral, $-7.85 \mathrm{~mm}$ ). The guide cannulas were maintained in position with dental cement supported by four jeweler's screws in the skull; dummy cannulas were kept in each guide at all times except during infusions. Immediately after surgery, rats were injected intramuscularly with $0.03 \mathrm{ml}$ each of a 100 $\mathrm{mg} / \mathrm{ml}$ solution of cephazolin and a $300 \mathrm{mg} / \mathrm{ml}$ solution of benacillin, and subcutaneously with $0.01 \mathrm{ml}$ of rimadyl. Rats were allowed $4-5 \mathrm{~d}$ to recover from surgery, during which they were handled and weighed daily. Rats were decapitated $\sim 1 \mathrm{~h}$ after the final test, and tissue sections were collected and stained for Nissl substance for verification of cannula placement using the Paxinos and Watson atlas (Paxinos and Watson, 1998). Ten rats were removed due to misplaced cannulas (three from the FGF2 group in Experiment 1, one from the Vehicle and three from the FGF2 groups in Experiment 2, and two from the FGF2 Reinstate and one from the Vehicle Reinstate groups in Experiment 3). See Figures 1C, 2D, and $3 D$ for cannula placements for rats included in the final analysis.

\section{Drug infusions}

Immediately after extinction training, the dummy cannulas were removed and 33 gauge injection cannulas, extending $1 \mathrm{~mm}$ below the tip of the guide cannula, were inserted for infusion of FGF2 (Bioscientific; R\&D Systems) or vehicle. FGF2 was dissolved in PBS containing $0.1 \%$ BSA and given in a dose of $200 \mathrm{ng}$ in $0.75 \mu \mathrm{l}$ infused in each hemisphere over 3 min using a microsyringe driven by a microinfusion pump (syringe pump SP101IZ; World Precision Instruments) via PE50 tubing. The injection cannulas were left in position for an additional $2 \mathrm{~min}$ before withdrawal. Although no previous studies have examined the effect of central infusions of FGF2 on memory, it has previously been demonstrated that $200 \mathrm{ng}$ of FGF2 infused intracerebroventricularly leads to observable behavioral effects in rats; thus, this was the basis for the chosen dose (Turner et al., 2008).

\section{Apparatus}

Two types of chambers were used to provide different contexts. One type was rectangular $(13.5 \mathrm{~cm}$ long $\times 9 \mathrm{~cm}$ wide $\times 9 \mathrm{~cm}$ high $)$, with the front wall, rear wall, and ceiling constructed of clear Plexiglas. The floor and side walls of these chambers consisted of $3 \mathrm{~mm}$ stainless-steel rods set 1 $\mathrm{cm}$ apart. These chambers were housed within wood cabinets so that external noise and visual stimulation were minimized. An infrared light was the sole source of illumination in these chambers (referred to as Context A hereafter).

The second type of chamber was rectangular $(30 \mathrm{~cm}$ long $\times 30 \mathrm{~cm}$ wide $\times 23 \mathrm{~cm}$ high) and wholly constructed of Plexiglas, with the exception of the grid floor that was the same as in the first type of chamber. The walls were transparent, except for two side walls that consisted of vertical black and white stripes ( $5 \mathrm{~cm}$ each). These chambers were housed in wood cabinets so that external noise and visual stimulation were minimized. A white LED and an infrared light were the sole sources of illumination in these chambers (referred to as Context B hereafter). Thus, these two sets of contexts differed primarily in terms of their size and in their visual features. In all experiments, rats were conditioned in Context $\mathrm{A}$ and extinguished and tested in Context B. In Experiment 2, all rats were additionally tested in Context A. In Experiment 3, the reinstatement procedure (or equivalent context exposure) took place in Context B.
The CS was a white noise; noise level in the chambers was increased by $8 \mathrm{~dB}$ when the CS was presented. The unconditioned stimulus (US) was a $0.6 \mathrm{~mA}, 1.0 \mathrm{~s}$ footshock. A computer controlled all presentations of the CS and the US.

\section{Procedure}

Handling and context preexposure. All rats were handled for 3-4 min each day for 3 consecutive days; after the rats were handled, on each day, they were placed in the conditioning chamber for $15 \mathrm{~min}$ (context preexposure).

Fear conditioning. Conditioning was identical in all experiments. On day 1 , rats received five pairings of the CS and US. Rats were placed in Context A, and after a 2 min adaptation period the CS was presented for $10 \mathrm{~s}$ and coterminated with the shock US. The intertrial interval (ITI) ranged from 85 to $135 \mathrm{~s}$, with a mean of $110 \mathrm{~s}$.

Extinction. On day 2 in Experiment 1, rats in the FGF2 and Vehicle groups received six, non-reinforced presentations of the CS. After a 2 min adaptation period, the CS was presented for $2 \mathrm{~min}$ with a $2 \mathrm{~min}$ ITI. We have previously demonstrated that these parameters lead to no difference in CS-elicited freezing at test between vehicle-treated rats that receive extinction training and those that do not (Ledgerwood et al., 2003, 2005). Thus, we chose these parameters to maximize the chance of detecting a facilitative effect of FGF2 on extinction recall (if one exists). Immediately after the last extinction trial, rats were infused (with FGF2 or vehicle) and then returned to their home cages. The FGF2 No Extinction group received equivalent context exposure but no CS presentations, immediately followed by FGF2 infusions. In Experiments 2 and 3, we attempted to equate the strength of extinction in the vehicle- and FGF2-treated rats; this needed to be done to allow for meaningful conclusions about the effect of FGF2 on renewal and reinstatement. Therefore, during extinction on day 2 in Experiments 2 and 3, the vehicle rats received 12 nonreinforced CS presentations ( 2 min CS with a 2 min ITI) while the FGF2 rats received 6 non-reinforced CS presentations. FGF2-treated rats remained in the extinction context for an extra 24 min after the last extinction trial; this equated the amount of exposure to the extinction context across the two conditions. Immediately after being removed from the extinction context, all rats were infused (with vehicle or FGF2). Vehicletreated rats also received an additional 12 extinction trials on day 3 in Experiments 2 and 3, while FGF2-treated rats received equivalent context exposure. No infusions occurred on day 3.

Reinstatement. On day 4 in Experiment 3 only, FGF2-treated rats and one group of vehicle-treated rats were returned to Context $B$ and received reinstatement. After a 2 min adaptation period, a single shock US (0.4 $\mathrm{mA}, 1 \mathrm{~s}$ ) was administered. Thirty to $60 \mathrm{~s}$ after the shock, rats were returned to their home cages. Vehicle-treated rats in the No Reinstate group were given an equivalent amount of exposure to Context $\mathrm{B}$, but not shocked.

Test. On day 3 in Experiment 1, day 4 in Experiment 2, and day 5 in Experiment 3, rats were tested for CS-elicited freezing. Pre-CS freezing was recorded for $2 \mathrm{~min}$ and then the CS was presented for $2 \mathrm{~min}$. See Table 1 for a summary of experimental procedures.

\section{Scoring and statistics}

Each animal was scored for freezing during extinction training and test. Freezing was scored by a time sampling procedure whereby each rat was scored every $3 \mathrm{~s}$ as freezing or not freezing. Freezing was defined as the absence of all movement other than that required for respiration (Fanselow, 1980). A percentage score was calculated for each animal to determine the proportion of total observations scored as freezing. The scorer was unaware of the condition of the rats. In Experiment 1, pre-CS freezing was analyzed using an independent-samples $t$ test (before extinction) and a one-way ANOVA (before test). In Experiment 2, pre-CS freezing was analyzed using an independent-samples $t$ test (before extinction training). In Experiment 3, pre-CS freezing was analyzed using a one-way ANOVA (before extinction) and pre-CS freezing before test was analyzed using a planned contrast comparing reinstated groups to the nonreinstated group, due to the a priori prediction that the reinstatement procedure would lead to higher levels of pre-CS freezing at test. In all experiments, within-session extinction training was analyzed using a 
Table 1. Experimental designs for Experiments 1, 2, and 3

\begin{tabular}{|c|c|c|c|c|c|}
\hline Experiment/group & Day 1 & Day 2 & Day 3 & Day 4 & Day 5 \\
\hline Experiment 1 & Conditioning & Extinction & Test & & \\
\hline Vehicle & $5 \mathrm{CS}+$ & $6 \mathrm{CS}-\rightarrow$ Vehicle & $\mathrm{CS}-$ & - & - \\
\hline FGF2 & $5 \mathrm{CS}+$ & $6 \mathrm{CS}-\rightarrow \mathrm{FGF2}$ & $\mathrm{CS}-$ & - & - \\
\hline Experiment 2 & Conditioning & Extinction day 1 & Extinction day 2 & Test & \\
\hline Vehicle & $5 \mathrm{CS}+$ & $12 \mathrm{CS}-\rightarrow$ Vehicle & $12 \mathrm{CS}-$ & $\mathrm{CS}-$ Context A/B & - \\
\hline FGF2 & $5 \mathrm{CS}+$ & $6 \mathrm{CS}-\rightarrow \mathrm{FGF2}$ & - & $\mathrm{CS}-$ Context A/B & - \\
\hline FGF2 Reinstate & $5 \mathrm{CS}+$ & $6 \mathrm{CS}-\rightarrow \mathrm{FGF} 2$ & - & Unsignaled US & $\mathrm{CS}-$ \\
\hline Vehicle No Reinstate & $5 \mathrm{CS}+$ & $12 \mathrm{CS}-\rightarrow$ Vehicle & $12 \mathrm{CS}-$ & - & $\mathrm{CS}-$ \\
\hline
\end{tabular}

mixed-design ANOVA. In Experiments 1 and 3, extinction recall was measured using a one-way ANOVA, and follow-up tests were conducted using Tukey's honestly significantly difference (HSD) test. In Experiment 2, pre-CS and CS-elicited freezing test data were analyzed using a repeated-measures ANOVA, and follow-up $t$ tests were conducted.

\section{Results \\ Experiment 1}

Experiment 1 investigated the effect of FGF2 when it was infused immediately after extinction training. There were no differences between levels of pre-CS freezing in FGF2- and vehicle-treated rats before extinction training $\left(t_{(15)}=0.37\right.$; $p=0.72)$. There was a significant effect of trial during extinction $\left(F_{(5,75)}=9.6 ; p<0.0001\right)$ and no effect of group or trial-by-group interaction (values of $F<1$ ), indicating that both groups exhibited comparable conditioning and rates of extinction (Fig. 1 $A$ ). This is to be expected given that drug infusion occurred after extinction training.

All groups exhibited comparable, low levels of pre-CS freezing before test $(F<1)$, but the groups differed in CS-elicited freezing during test $\left(F_{(2,22)}=11.94 ; p<0.0001 ;\right.$ Fig. $\left.1 B\right)$. This difference was due to FGF2-treated rats that received extinction exhibiting significantly lower levels of freezing compared with both the FGF2-treated rats that did not receive extinction $(p<0.0001)$ and the vehicle-treated rats that did receive extinction $(p=$ $0.004)$. The latter two groups did not differ from one another $(p=0.55)$. In sum, Experiment 1 demonstrated that postextinction training, intra-BLA infusions of FGF2 facilitate extinction recall at test. This effect was not solely due to nonspecific effects of FGF2 on anxiety or motoric activity because FGF2 did not reduce freezing at test in nonextinguished rats. It should be noted that vehicle-treated rats that received extinction exhibited comparable levels of freezing at test to FGF2-treated rats that did not receive extinction. This indicates that vehicle-treated rats exhibited almost complete recovery of fear at test, which is consistent with previous studies that used these parameters (Ledgerwood et al., 2003, 2005).

\section{Experiment 2}

In this experiment, the effect of postextinction training intraBLA infusions of FGF2 on renewal was examined using a withinsubjects design. In an attempt to equate the strength of extinction between the two groups, vehicle-treated rats received four times the amount of extinction compared to FGF2-treated rats (24 extinction trials over days 2 and 3). FGF2-treated rats received six extinction trials on day 2 and equivalent context exposure on days 2 and 3 to equate the amount of time spent in the extinction context between the two groups. All rats were tested for CSelicited freezing in the extinction training context and in the orig- inal fear conditioning context on day 4 (tests were separated by $\sim 4$ h and the order was counterbalanced within groups).

There were no differences between groups in pre-CS freezing before extinction training $\left(t_{(13)}=1.2 ; p=0.29\right)$. There was a significant effect of trial during the first six extinction trials $\left(F_{(5,65)}=5.41 ; p=0.006\right)$, and no effect of group or trial-bygroup interaction (values of $F<1$ ), indicating that both groups exhibited comparable levels of conditioning and rates of extinction (Fig. $2 A$ ). There was a significant effect of trial during extinction on day $2\left(F_{(11,77)}=9.45 ; p<0.0001\right)$, indicating that vehicle-treated rats continued to extinguish on the second day of extinction training (Fig. $2 B$ ).

Analysis of pre-CS elicited freezing before test in Context A and Context $\mathrm{B}$ revealed a significant effect of context $\left(F_{(1,11)}=\right.$ 7.06; $p=0.022$ ), but no effects of group or test order, and no interactions of group or test order ( $\operatorname{smallest} p=0.103$; Fig. $2 C$ ). This indicates that all rats exhibited comparable increases in pre-CS freezing when tested in Context A (i.e., the original fear conditioning context), regardless of drug condition or order of test. Analysis of CS-elicited freezing (Fig. 2C) during test in Context $\mathrm{A}$ and Context $\mathrm{B}$ revealed a significant within-subjects effect of context $\left(F_{(1,11)}=68.76 ; p<0.0001\right)$. Furthermore, there was a significant between-subjects effect of drug $\left(F_{(1,11)}=6.23 ; p=\right.$ $0.03)$, and a significant drug-by-context interaction $\left(F_{(1,11)}=\right.$ $15.14 ; p=0.03)$. There were no effects of test order, and no interactions between test order and any other factor (smallest $p=$ 0.28). A comparison of freezing in Context A versus freezing in Context $\mathrm{B}$ for each drug group indicated that both drug groups exhibited higher CS-elicited freezing in Context A compared with Context B (for FGF2-treated rats, $t_{(6)}=2.95, p=0.026$; for vehicle-treated rats, $t_{(7)}=26.5, p<0.0001$ ), demonstrating that both groups exhibited some renewal of fear. However, FGF2treated rats exhibited significantly less CS-elicited freezing than vehicle rats when tested in Context $\mathrm{A}\left(t_{(13)}=4.21 ; p=0.001\right)$, indicating that FGF2-treated rats exhibited significantly attenuated renewal. Finally, FGF2- and vehicle-treated rats exhibited comparable levels of CS-elicited freezing when tested in Context $\mathrm{B}$, indicating that giving vehicle-treated rats four times the amount of extinction training as FGF2-treated rats successfully equated the strength of the extinction memory when rats were tested in the same context as extinction training $\left(t_{(13)}=0.75 ; p=\right.$ $0.47)$. These results remained statistically significant when corrected for multiple comparisons (all corrected values of $p<$ 0.0125 ). Thus, this experiment demonstrated that vehicle-treated rats require four times the amount of extinction training over $2 \mathrm{~d}$ to exhibit equivalent extinction recall to FGF2-treated rats. Furthermore, this experiment demonstrated that FGF2-treated rats exhibit significantly attenuated renewal of fear compared with 

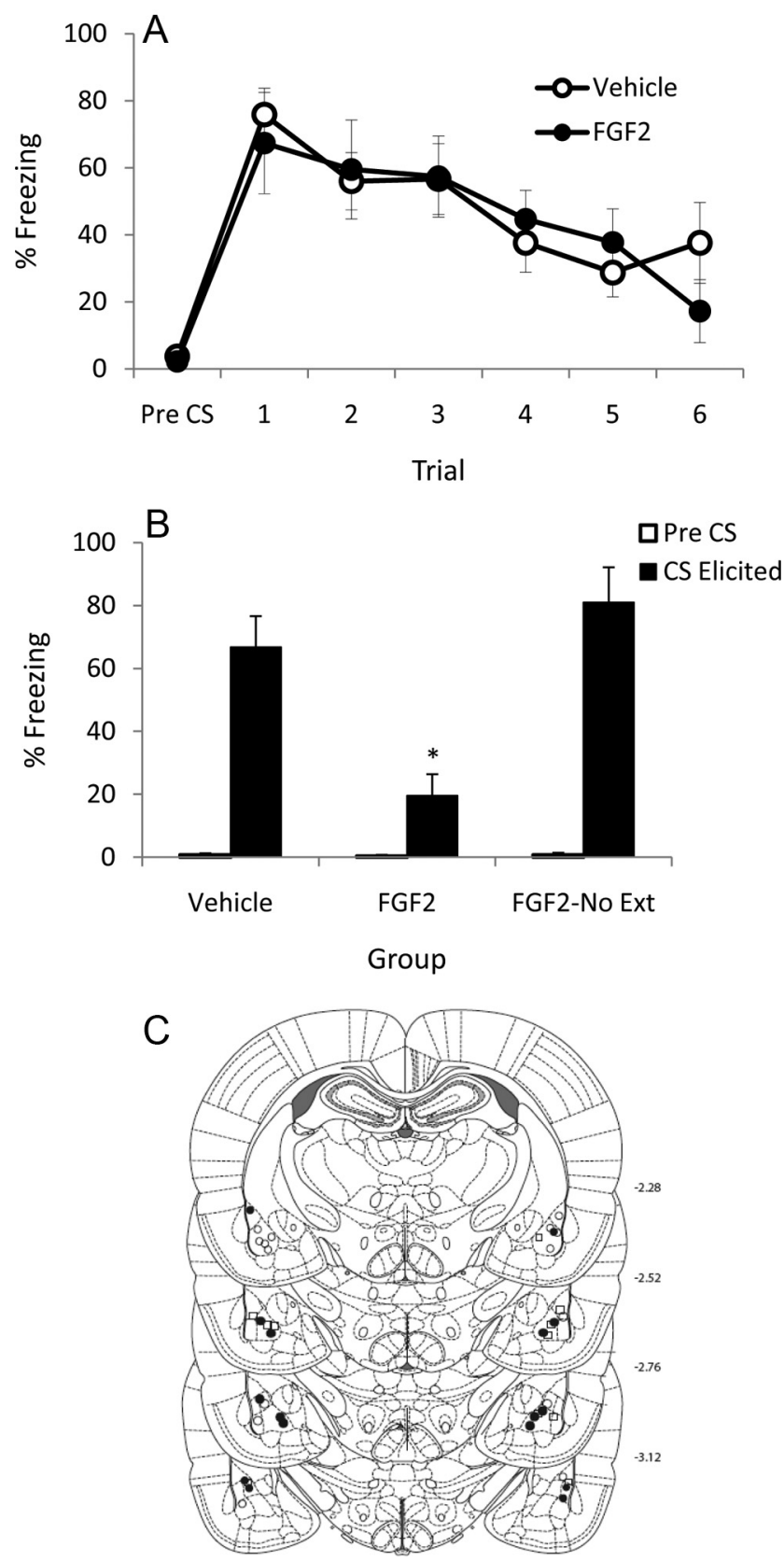

Figure 1. $A$, Mean (+SEM) pre-CS and CS-elicited freezing during extinction training in Experiment 1.B.Mean (+SEM) pre-CS and CS-elicited freezing during test in Experiment 1. The asterisk $\left({ }^{*}\right)$ indicates a significant difference between groups in (S-elicited freezing. $C$, Cannula placements for all rats included in the final analysis of Experiment 1. ๑, FGF2 $(n=8) ; \bigcirc$, Vehicle $(n=8) ; \square$, FGF2-No Ext $(n=8)$.

vehicle-treated rats, even when vehicle-treated rats receive four times the amount of extinction training.

\section{Experiment 3}

This experiment examined the effect of postextinction training intra-BLA infusions of FGF2 on reinstatement, or stressprecipitated relapse. Again, vehicle-treated rats received four times the amount of extinction to equate the strength of extinction between vehicle- and FGF2-treated rats. On day 4, one-half of the vehicle-treated rats and all of the FGF2-treated rats received a mild unsignaled shock in the extinction context. The other one-half of vehicle-treated rats received equivalent context
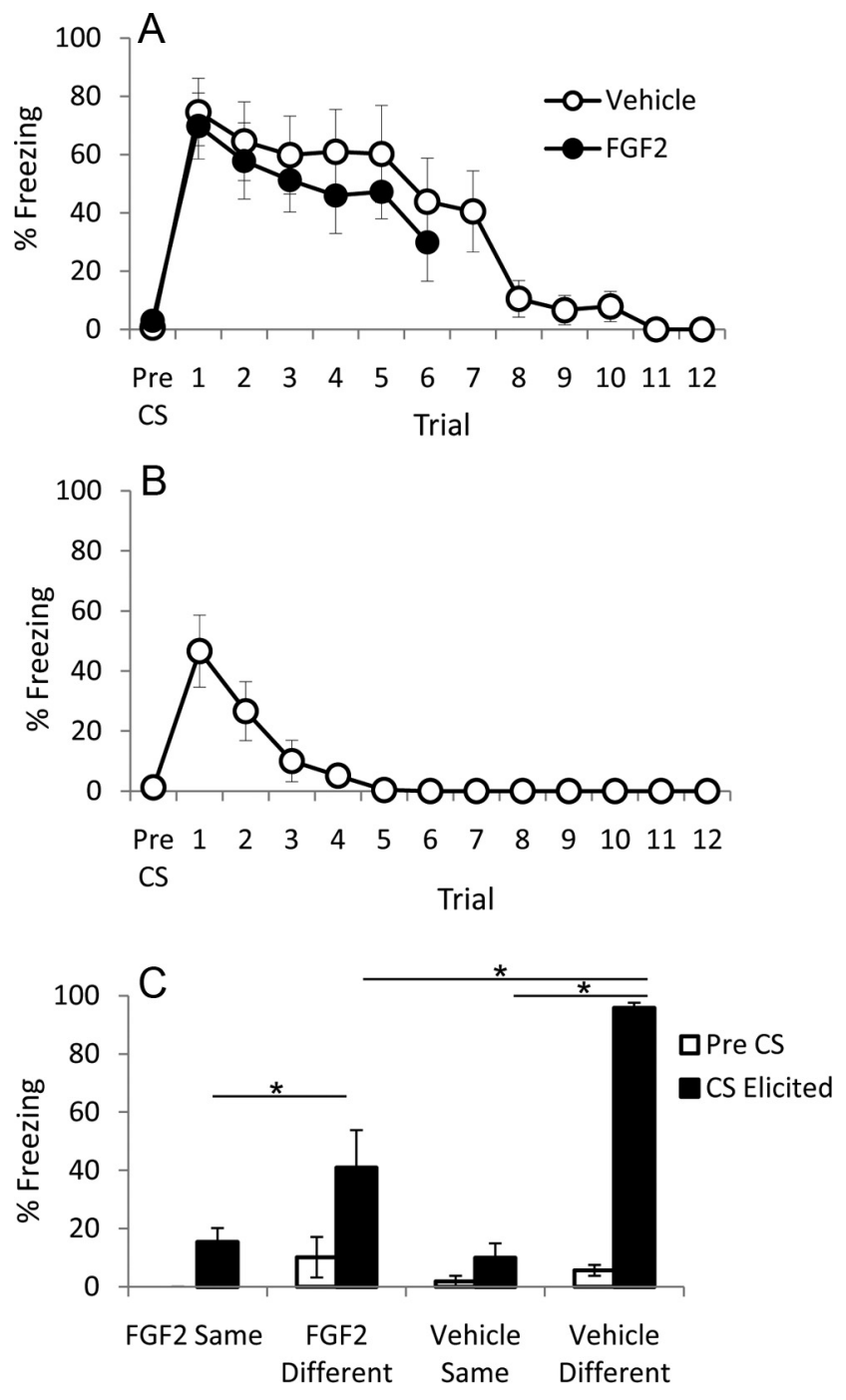

Group and Test Context

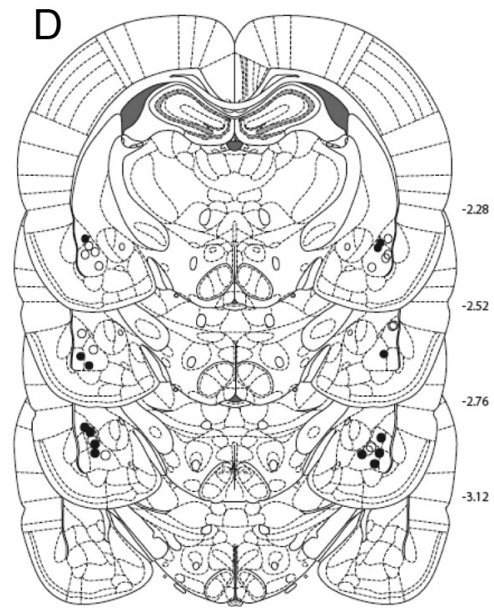

Figure 2. $A$, Mean (+SEM) pre-CS and CS-elicited freezing during extinction training in Experiment 2. B, Mean (+SEM) pre-CS and CS-elicited freezing during day 2 of extinction training in Experiment 2 for vehicle-treated rats. C, Mean (+SEM) pre-CS and CS-elicited freezing during test in Experiment 2 in Context A ("different") and Context B ("same"). The asterisk $\left.{ }^{*}\right)$ indicates a significant difference between groups in CS-elicited freezing; see text for details on significant differences in pre-CS freezing. $\boldsymbol{D}$, Cannula placements for all rats included in the final analysis of Experiment 2. $\mathrm{O}$, FGF2 $(n=7) ; \bigcirc$, Vehicle $(n=8)$. 
exposure, but did not receive shock. All rats were tested for CSelicited freezing in the extinction training context on day 5.

There were no differences in pre-CS freezing before extinction training $\left(F_{(2,19)}=1.08 ; p=0.36\right.$; Fig. $\left.3 A\right)$. Furthermore, an analysis of the first six trials of extinction training revealed a significant effect of trial $\left(F_{(5,85)}=3.12 ; p=0.013\right.$; Fig. $\left.3 A\right)$, but no effect of group and no trial-by-group interaction $(F \mathbf{s}<1)$, demonstrating that there were no group differences in levels of conditioning or rates of extinction. An analysis of CS-elicited freezing during the second six trials of extinction training (only administered to vehicle-treated rats) revealed a significant effect of trial $\left(F_{(5,60)}=\right.$ $4.44 ; p=0.002)$, but no effect of group $\left(F_{(1,12)}=2.45 ; p=0.14\right)$ and no trial-by-group interaction $\left(F_{(5,60)}=1.77 ; p=0.13\right)$, indicating that vehicle-treated rats continued to extinguish comparably during the next six trials, regardless of whether they were to-be-reinstated or not. Furthermore, analysis of CS-elicited freezing during the 12 trials of extinction training on day 2 (only administered to vehicle-treated rats) revealed a significant effect of trial $\left(F_{(11,132)}=9.21 ; p=0.03\right)$, but no effect of group $\left(F_{(1,12)}=2.27 ; p=\right.$ $0.15)$ and no trial-by-group interaction $\left(F_{(11,132)}=1.75 ; p=0.20\right)$, indicating that these groups continued to extinguish comparably during extinction training on day 2 (Fig. $3 B$ ).

Both FGF2- and vehicle-treated rats given the pretest shock exhibited slightly higher levels of pre-CS freezing than did those rats not given the pretest shock $\left(t_{(17)}=2.24 ; p=0.039\right.$; Fig. $\left.3 C\right)$. The groups also differed in levels of CS-elicited freezing $\left(F_{(2,19)}=\right.$ $7.75 ; p=0.004$; Fig. $3 C$ ), and this was due to vehicle-treated rats that received the pretest shock freezing more to the CS than either the non-reinstated vehicle-treated rats $(p=0.04)$ or the FGF2treated rats that did receive reinstatement $(p=0.03)$. The latter two groups did not differ from one another $(p=0.72)$. Thus, Experiment 3 demonstrated that intra-BLA infusions of FGF2 immediately after extinction training reduced shock-induced reinstatement of fear.

To confirm that the FGF2 effect was dependent on it being infused in the BLA, we conducted a post hoc analysis of the six rats from Experiments 1 and 2 (only using freezing scores from rats that were tested in Context B first) that were excluded due to misplacement of cannulas in the regions surrounding the BLA. We compared this group to the groups in Experiment 1. A oneway ANOVA indicated no difference between groups in levels of pre-CS freezing $(F<1)$, and a significant difference in levels of CS-elicited freezing at test $\left(F_{(3,28)}=7.462 ; p=0.001\right)$. Follow-up tests using Tukey's HSD demonstrated that misplaced FGF2treated rats exhibited significantly higher CS-elicited freezing compared with FGF2-treated rats that received extinction (mean CS-elicited freezing of misplaced FGF2 rats, 62.83; SEM, 12.65; $p=0.03$ ), while exhibiting comparable CS-elicited freezing as vehicle-treated rats and FGF2-treated rats that did not receive extinction training (smallest $p=0.62$ ). This demonstrates that targeted infusion of FGF2 into the BLA is required to enhance extinction.

\section{Discussion}

The principal findings of these experiments are as follows: (1) intra-BLA infusions of FGF2 enhance extinction recall when administered immediately after extinction training (Experiment 1 ), (2) vehicle-treated rats require four times the amount of extinction training over $2 \mathrm{~d}$ to exhibit comparable extinction recall to FGF2-treated rats (Experiment 2, replicated in Experiment 3), (3) intra-BLA infusions of FGF2 immediately after extinction training significantly attenuates renewal in a within-subjects design (Experiment 2), and (4) intra-BLA infusions of FGF2 immediately after
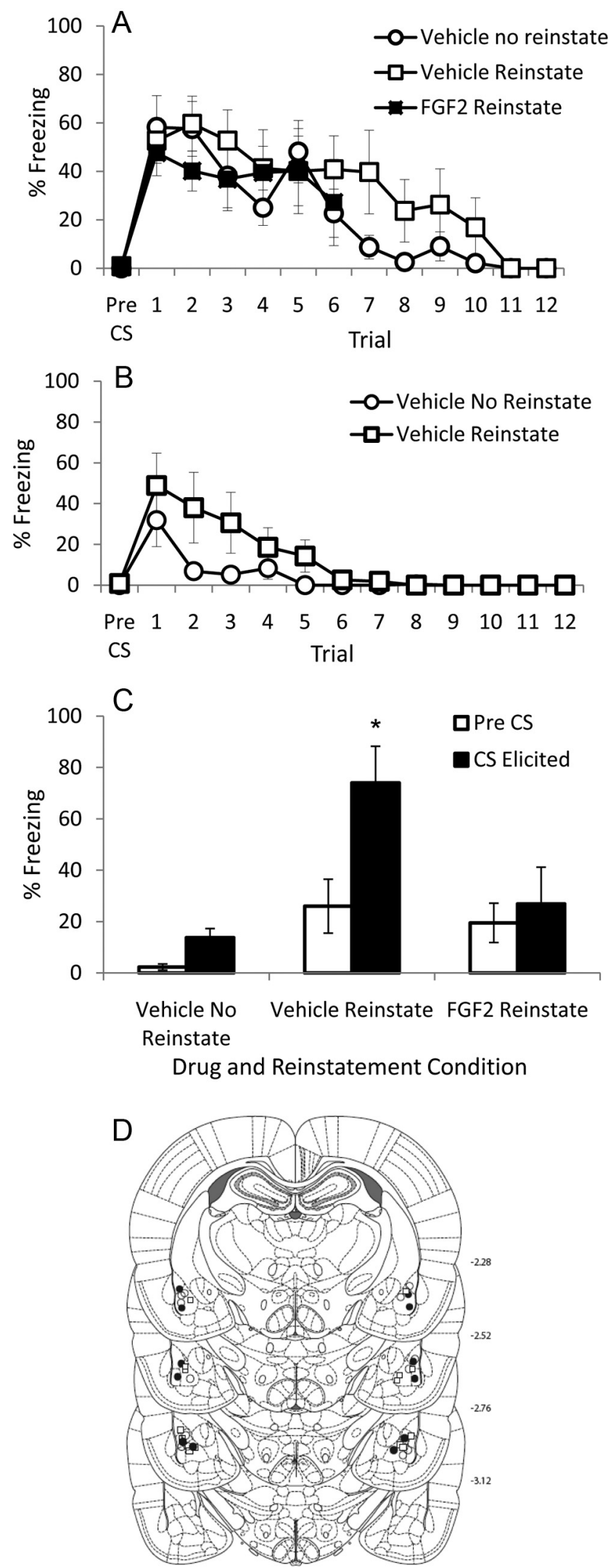

Figure 3. $A$, Mean (+SEM) pre-CS and CS-elicited freezing during extinction training in Experiment 3 . $\boldsymbol{B}$, Mean (+SEM) pre-CS and CS-elicited freezing during day 2 of extinction training in Experiment 3 for vehicle-treated rats. C, Mean (+SEM) pre-CSandCS-elicited freezing during test in Experiment 3. The asterisk $\left(^{*}\right)$ indicates a significant difference between groups in CS-elicited freezing; see text for details on significant differences in pre-CS freezing. $D$, Cannula placements for all rats included in the final analysis of Experiment 3. FGF2 Reinstate $(n=6) ; \bigcirc$, Vehicle No Reinstate $(n=7) ; \square$, Vehicle Reinstate $(n=7)$. 
extinction training abolishes reinstatement (Experiment 3). These experiments show that the effects of FGF2 on extinction recall, and relapse, are comparable in $\mathrm{P} 23$ and adult rats. This agrees with other research that suggests that the neural, molecular, and behavioral characteristics of extinction are similar in P23 and adult rats (for review, see Kim and Richardson, 2010).

These results provide at least two insights into the mechanisms by which FGF2 enhances extinction. First, these results suggest that FGF2 acts centrally via the BLA (at least in part) to enhance extinction and reduce subsequent relapse, as intra-BLA infusions of FGF2 produce comparable results with those seen with systemic injections (Graham and Richardson, 2009, 2010). This conclusion is supported by reports that FGF2 crosses the blood-brain barrier (Cuevas et al., 1996) and that peripherally administered FGF2 has central effects (e.g., increased neurogenesis) (Wagner et al., 1999). Furthermore, this is consistent with research showing that the amygdala is a key structure in the neural circuitry of extinction (Quirk and Mueller, 2008), and with findings in rodents that the amygdala contains high concentrations of FGF2 and Fgfr 1 (the main receptor to which FGF2 binds) expression (Gonzalez et al., 1995). Although infusions of FGF2 into the BLA are equivalent to its effects when administered systemically, it is possible that systemically administered FGF2 may also act in other structures implicated in extinction, such as the ventromedial prefrontal cortex and/or the hippocampus. Indeed, FGF2 and Fgfr1 are highly expressed in most regions of the hippocampus, including the dentate gyrus, CA1, CA2, and CA3. However, FGF2 and Fgfrl have only been shown to be expressed in the ventrolateral prefrontal cortex, rather than the infralimbic region that is more commonly implicated in extinction memories (Gonzalez et al., 1995).

Second, it was demonstrated that in order to reduce test freezing below the level of that expressed by vehicle-treated rats, FGF2 must be combined with extinction. Without comparing FGF2nonextinguished rats to a group of vehicle-treated rats that also did not receive extinction, it cannot be claimed with certainty that FGF2 has no nonspecific effects on fear expression, anxiety, or motor response. However, the finding that FGF2-treated rats exhibited conditioning to the context in which they received an unsignaled footshock in Experiment 3 demonstrates that FGF2 infusion does not impair the ability to acquire or express fear. Furthermore, given that FGF2 No Extinction rats exhibited comparable freezing during test to that exhibited by vehicle-treated rats during the first extinction training trial, it is likely that the observed effects are due to FGF2 altering extinction in some way. It is increasingly accepted that fear extinction involves both new learning and unlearning, or erasure components, as relapse is rarely complete. It has been suggested that the molecular signature of unlearning may be depotentiation, a type of synaptic plasticity that reverses long-term potentiation at the synapse (Lin et al., 2003). Depotentiation is associated with increases in growth factors (Yuzaki et al., 1994) and is partly mediated by calcineurin, a molecule that has been demonstrated to be modulated by FGF2 (Boxer et al., 1999). Therefore, it is possible that FGF2 may upregulate the unlearning component of extinction via its effects on calcineurin-mediated depotentiation. This is consistent with our findings that FGF2 attenuates or in some cases abolishes relapse. Also consistent with these findings is the possibility that FGF2 may disrupt the reconsolidation of the original fear memory when it is reactivated during the extinction procedure. However, this is inconsistent with our previous finding that, in P23 rats, FGF2 was ineffective in enhancing extinction following a short extinction procedure (which is more akin to memory reactiva- tion than the extinction procedure used in the present experiments) (Graham and Richardson, 2009).

The partial NMDA receptor agonist D-cycloserine (DCS) has also been shown to be effective at facilitating extinction of learned fear (for review, see Davis et al., 2006). Similar to FGF2, DCS reduces expression of learned fear only when given together with extinction and is effective when administered systemically or when infused into the BLA (Walker et al., 2002; Ledgerwood et al., 2003). In addition, animals given DCS are resistant to reinstatement of fear (Ledgerwood et al., 2004; Bertotto et al., 2006). However, DCS-treated animals have been reported to exhibit the same renewal of extinguished fear as vehicle-treated animals (Woods and Bouton, 2006), in contrast to our present and previous findings, suggesting that FGF2 and DCS may modulate extinction via different mechanisms. For example, it is possible that DCS may enhance inhibitory learning about the extinction context, such that DCS-treated rats have a stronger representation of the extinction context as a safety cue (Woods and Bouton, 2006). If so, this would block subsequent conditioning to the extinction context that occurs during the reinstatement procedure and, in turn reduce reinstatement. However, consistent with the finding by Woods and Bouton (2006), this would not prevent renewal of fear when rats are tested in a different context. Clearly, FGF2 does not enhance extinction by facilitating inhibitory learning about the extinction context because (1) FGF2-treated rats exhibit markedly reduced renewal and (2) FGF2-treated rats exhibit comparable levels of conditioning to the extinction context following reinstatement as do vehicle-treated rats. If it is the case that FGF2 and DCS enhance extinction via different mechanisms, then it is also possible that FGF2 facilitation of extinction may not be subject to the same limitations as is the DCS facilitation of extinction (e.g., repeated exposure to DCS produces a temporary loss of its effectiveness) (Parnas et al., 2005), although this remains to be tested.

FGF2 is not the only neurotrophic factor that has been implicated in extinction. Brain-derived neurotrophic factor (BDNF) mRNA is elevated in the BLA following extinction of conditioned fear, and disruption of BNDF signaling via lentiviral infection disrupts long-term retention of extinction (Chhatwal et al., 2006). It was also recently demonstrated that intra-infralimbic (IL) prefrontal cortex infusions of BDNF reduced expression of conditioned fear the day after infusions, an effect that occurred even in the absence of extinction training (Peters et al., 2010). BDNF-treated rats in that study exhibited comparable reinstatement of fear to vehicle-treated rats, demonstrating that BDNF did not merely reduce freezing behavior, and prompting the suggestion that intra-IL infusion of BDNF "substituted" for extinction. Despite there being much overlap between the roles of FGF2 and BDNF in the CNS, it appears that the two neurotrophic factors may modulate fear extinction via different mechanisms. It is clear that FGF2, unlike BDNF, does not substitute for extinction (as it is ineffective when administered without extinction training), and moreover, FGF2 appears to change the quality of extinction and renders rats less susceptible to relapse, in contrast to BDNF. However, it is also possible that BDNF and FGF2 may have different effects on extinction depending on which structure within the neural circuitry of extinction they are infused, and future studies should investigate this.

Overall, these findings support the growing body of evidence that FGF2 is a powerful modulator of extinction. Particularly striking in the present findings is that extinction combined with FGF2 is as effective as four times the amount of extinction without FGF2. This increased efficiency, combined with the effect of 
FGF2 on relapse of fear, make FGF2 an attractive candidate for a novel pharmacological adjunct that may improve existing treatments for anxiety across different age groups.

\section{References}

Bertotto ME, Bustos SG, Molina VA, Martijena ID (2006) Influence of ethanol withdrawal on fear memory: effect of D-cycloserine. Neuroscience 142:979-990.

Boxer AL, Moreno H, Rudy B, Ziff EB (1999) FGF-2 potentiates $\mathrm{Ca}^{2+}$ dependent inactivation of NMDA receptor currents in hippocampal neurons. J Neurophysiol 82:3367-3377.

Chhatwal JP, Stanek-Rattiner L, Davis M, Ressler KJ (2006) Amygdala BDNF signaling is required for consolidation but not encoding of extinction. Nat Neurosci 9:870-872.

Cuevas P, Fernández-Ayerdi A, Carceller F, Colin S, Mascarelli F, MuñozWillery I, Giménez-Gallego G (1996) Central nervous system distribution of fibroblast growth factor injected into the blood stream. Neurol Res 18:267-272.

Davis M, Ressler K, Rothbaum BO, Richardson R (2006) Effects of D-cycloserine on extinction: translation from preclinical to clinical work. Biol Psychiatry 60:369-375.

Fanselow MS (1980) Conditional and unconditional components of postshock freezing. Pavlov J Biol Sci 15:177-182.

Gonzalez AM, Berry M, Maher PA, Logan A, Baird A (1995) A comprehensive analysis of the distribution of FGF-2 and FGFR1 in the rat brain. Brain Res 701:201-226.

Graham BM, Richardson R (2009) Acute systemic fibroblast growth factor-2 enhances long-term extinction of fear and reduces reinstatement in rats. Neuropsychopharmacology 34:1875-1882.

Graham BM, Richardson R (2010) Fibroblast growth factor-2 enhances extinction and prevents renewal of conditioned fear. Neuropsychopharmacology 35:1348-1355.

Graham BM, Richardson R (2011) Fibroblast growth factor-2 alters the nature of extinction. Learn Mem 18:80-84.

Graham BM, Langton JM, Richardson R (2011) Pharmacological enhancement of fear reduction: preclinical models. $\mathrm{Br} \mathrm{J}$ Pharmacol. Advance online publication. Retrieved August 23, 2011. doi:10.1111/j.1476-5381.2010.01175.x.

Hofmann SG, Smits JA (2008) Cognitive-behavioural therapy for adult anxiety disorders: a meta-analysis of randomized placebo-controlled trials. J Clin Psychiatry 69:621-632.

Kim JH, Richardson R (2010) New findings on extinction of conditioned fear early in development: theoretical and clinical implications. Biol Psychiatry 67:297-303.

Ledgerwood L, Richardson R, Cranney J (2003) Effects of D-cycloserine on extinction of conditioned freezing. Behav Neurosci 117:341-349.
Ledgerwood L, Richardson R, Cranney J (2004) D-Cycloserine and the facilitation of extinction of conditioned fear: consequences for reinstatement. Behav Neurosci 118:505-513.

Ledgerwood L, Richardson R, Cranney J (2005) D-Cycloserine facilitates extinction of learned fear: effects on reacquisition and generalized extinction. Biol Psychiatry 57:841-847.

LeDoux JE (2000) Emotion circuits in the brain. Annu Rev Neurosci 23: $155-184$.

Lin CH, Lee CC, Gean PW (2003) Involvement of a calcineurin cascade in amygdala depotentiation and quenching of fear memory. Mol Pharmacol 63:44-52.

McNally RJ (2007) Mechanisms of exposure therapy: how neuroscience can improve psychological treatments for anxiety disorders. Clin Psychol Rev 27:750-759.

Monfils MH, Driscoll I, Melvin NR, Kolb B (2006) Differential expression of basic fibroblast growth factor- 2 in the developing rat brain. Neuroscience 141:213-221.

Parnas AS, Weber M, Richardson R (2005) Effects of multiple exposures to D-cycloserine on extinction of conditioned fear in rats. Neurobiol Learn Mem 83:224-231.

Paxinos G, Watson C (1998) The rat brain in stereotaxic coordinates. New York: Academic.

Peters J, Dieppa-Perea LM, Melendez LM, Quirk GJ (2010) Induction of fear extinction with hippocampal-infralimbic BDNF. Science 328: $1288-1290$.

Quirk GJ, Mueller D (2008) Neural mechanisms of extinction learning and retrieval. Neuropsychopharmacology 33:56-72.

Rai KS, Hattiangady B, Shetty AK (2007) Enhanced production and dendritic growth of new dentate granule cells in the middle-aged hippocampus following intracerebroventricular FGF-2 infusions. Eur J Neurosci 26:1765-1779.

Turner CA, Gula EL, Taylor LP, Watson SJ, Akil H (2008) Antidepressantlike effects of intracerebroventricular FGF2 in rats. Brain Res 1224:63-68.

Wagner JP, Black IB, DiCicco-Bloom E (1999) Stimulation of neonatal and adult brain neurogenesis by subcutaneous injection of basic fibroblast growth factor. J Neurosci 19:6006-6016.

Walker DL, Ressler KJ, Lu KT, Davis M (2002) Facilitation of conditioned fear extinction by systemic administration or intraamygdala infusions of $\mathrm{D}$-cycloserine as assessed with fear potentiated startle in rats. J Neurosci 22:2343-2351.

Woods AM, Bouton ME (2006) D-Cycloserine facilitates extinction but does not eliminate renewal of the conditioned emotional response. Behav Neurosci 120:1159-1162.

Yuzaki M, Furuichi T, Mikoshiba K, Kagawa Y (1994) A stimulus paradigm inducing long-term desensitization of AMPA receptors evokes a specific increase in BDNF mRNA in cerebellar slices. Learn Mem 1:230-242. 